\title{
Effects of a computerised protocol management system on ordering of clinical tests
}

\author{
P G Nightingale, M Peters, D Mutimer, J M Neuberger
}

\begin{abstract}
Objective-To assess the effects of a computerised protocol management system on the number, cost, and appropriateness of laboratory investigations requested.
\end{abstract}

Design-A before and after intervention. Setting-A supraregional liver unit in a teaching hospital.

Patients-1487 consecutive patients admitted during 1990 and 1991 (one year before and one year after introduction of the system).

Intervention-Introduction of a computerised protocol management system on 1 January 1991.

Main measures-The number and cost of clinical chemistry tests requested per patient day.

Results-The total number of clinical chemistry tests requested per patient day by the unit declined $17 \% \quad(p<0.001$, Student's $t$ test) and of out of hours tests requested per patient day from 0.31 to $0 \cdot 16,48 \%$ (p < 0.001; Mann-Whitney $U$ test), resulting in a $28 \%$ reduction $(p<0.001)$ in direct laboratory expenditure per patientday. Overall, the number of tests per admission decreased by $24 \%(p<0.001$; Mann-Whitney U test).

Conclusion-Use of the computerised protocol management system resulted in closer compliance with the protocols and a significant reduction in the overall level of requesting.

Implications-Although similar systems need to be tested in other clinical settings, computerised protocol management systems may be important in providing appropriate and cost effective health care.

(Quality in Health Care 1994;3:23-28)

Wolfson Computer

Laboratory,

Queen Elizabeth

Medical Centre,

Birmingham B15 2TH

$P$ G Nightingale,

statistician

M Peters, director

Liver Unit,

The Queen Elizabeth

Hospital,

Birmingham B15 2TH

D Mutimer, research

registrar

J M Neuberger,

consultant physician

Correspondence to:

Dr Nightingale

Accepted for publication

26 April 1994

\section{Introduction}

Although often controversial, ${ }^{1}$ protocols are essentially just formalised approaches to managing patients and as such should provide a way of improving the appropriateness of investigations performed. ${ }^{2}$ However, where protocols have been devised they have not always been adhered to, owing, among other reasons, to the continual need to train new staff in their use and, perhaps more importantly, to the difficulty in applying routinely several, sometimes quite complex, rules simultaneously.

The liver unit at this hospital is a specialist unit with a particular interest in liver failure and transplantation and is a secondary and tertiary referral centre for patients with serious liver disease. It is a significant user of laboratory resources, requesting 63871 clinical chemistry tests in $1990(10 \cdot 1 \%$ of the total workload of the clinical chemistry laboratory at this hospital), and is the main source of requests for many of the more complex and expensive investigations in the laboratory's repertoire. In 1989 the unit had devised a set of laboratory investigation protocols in an attempt to improve the appropriateness of its laboratory requesting. However, the complexity of some of these protocols (see appendix for examples) made it extremely difficult for house officers to comply with them exactly when requesting investigations.

It was this complexity that prompted us to design a computerised protocol management system for the unit. This decision support system expressed the unit's existing investigation protocols (see appendix) in the form of rules based on information regarding each patient's current clinical state (for example, assessment, transplant, etc) and latest pathological results. By evaluating these rules, the system proposes the investigations to be performed on each patient on the following day. Once these proposals have been authorised, with modification if required, labels for request cards and specimen tubes are printed.

The computerised protocol management system was introduced into the unit on 1 January 1991. A study was undertaken to assess whether the routine use of such a system had any effect on the time spent by house officers requesting laboratory investigations and inquiring about specimens or results; the appropriate use of laboratory services; and the training of the junior clinical staff. This study was not concerned primarily with the effects of the protocols themselves but with the effects of using a computer system to implement them. After introduction of the system the time house officers spent requesting laboratory investigations and inquiring about specimens or results was reduced $59 \% .^{3}$ A broad comparison of two three-month periods before and after the introduction disclosed significant decreases also in the number of clinical chemistry tests requested for both transplant and non-transplant recipients (10\% and $29 \%$ respectively).

In this paper we report in detail the clinical chemistry requesting of the unit in the year before and the year after the introduction of the system. We determine the effect the system had on the requesting behaviour of the house officers and attempt to assess whether the system trained them to request more appropriately. 


\section{Methods}

PROTOCOLS

The investigation protocols (see appendix) for each category of inpatient seen in the unit had been developed by the senior clinicians in an effort to improve the appropriateness of the investigations requested. They consisted of a combination of static and dynamic rules: static rules are those which propose investigations for any patient with a certain classification for a specified number of days (for example, "propose liver function tests for any transplant recipient on each of the first 14 days") dynamic rules are those which use the results of previous analyses to determine which investigations to propose (for example, "if a transplant recipient's latest potassium result is $>5 \cdot 1$, propose urea and electrolyte tests for that patient tomorrow"). Thus the investigations proposed for a patient within a particular category will consist of the "core" set of investigations proposed for all patients in that category plus any additional proposals triggered by the results of previous analyses.

Before the introduction of the protocol management system the complexity of these protocols had prevented them from being implemented properly. In particular, it was known that for many patients investigations in the assessment protocol (see appendix) were frequently omitted and that some of the more common investigations were repeated more often than necessary.

When introduced into the unit, the computerised protocol management system had been using the existing protocols. Four months after a modification was made, as follows. Previously, a plasma calcium measurement was automatically requested whenever liver function tests (albumin, alkaline phosphatase, aspartate aminotransferase, and total bilirubin tests) were requested; subsequently a plasma calcium measurement had to be requested specifically. No other major changes were made in the year after introduction of the system, although the original "assessment" classification has since been replaced with several disease-specific classifications (for example, "Wilson's disease assessment"), each with their own investigation protocol.

COMPUTERISED PROTOCOL MANAGEMENT

The system ${ }^{4} 5$ was written in MUMPS and was implemented on a 386 personal computer linked to the hospital patient administration system and to the clinical chemistry laboratory's computer system in order to receive patient details and test results.

For each admission to the unit the house officer must enter into the system the clinical categories applicable to that patient. He or she must also alter the classifications of current inpatients when necessary (for example, if a patient undergoes transplantation). Classifying a patient takes only seconds but is crucial to the proper functioning of the system, as these clinical categories determine which protocols are applied to each patient. The system uses the information entered by the house officer, together with the latest available results (obtained automatically from the appropriate laboratory computer system), to propose the investigations to be performed on each patient on the following day. For any patient the house officer may view on one screen page or more all the investigations proposed for that patient on the following day and can accept or modify these proposals as required. This is usually done at the end of the normal working day (so that as many results as possible have been received from the laboratory). Once the house officer has accepted or modified the proposals for each patient, the request and specimen labels to be used the following day can be printed in one batch. It is thus in the house officer's own interest to ensure that patients are properly classified so that the appropriate investigations are proposed automatically and the number of modifications minimised.

New house officers are trained in the use of the system by their senior colleagues within the unit, a typical training session lasting about an hour. The management system contains extensive on-screen help, obviating the need for staff to be issued with handbooks, although a user manual is available for reference. Displayed on the terminal is a telephone number to ring for hardware and software support (which is available during normal working hours). Further training is gained by discussion with colleagues after hands-on use of the system. Although additional training sessions are available on request, they have as yet not proved to be necessary.

FEEDBACK

The system automatically records any result received from the clinical chemistry laboratory's computer for a patient in the unit and also determines whether this investigation was proposed by the protocol management system. With this information a monthly summary of the investigations performed on these patients is provided to the consultants, showing the total number of requests for each investigation, for patients in each clinical category, and for each day of the month. For each of these totals the proportion of requests proposed by the protocols is also shown, giving some measures of "protocol performance." This information is used by the consultants as a basis for regular discussion with the junior staff regarding possible amendments to their current practice or to the protocols (for example, expanding the number of patient classifications, adding further dynamic rules and modifying the existing rules, etc), or both. Thus the performance of the protocols is constantly being reviewed in the light of the latest information, and the original protocols have now been considerably enhanced.

SETTING

The study involved 1487 consecutive patients admitted to the unit in the year before and the year after the introduction of the computerised protocol management system (calendar years 1990 and 1991 respectively). Details of all these patients, such as their hospital registration 
number, date of admission, date of discharge, and clinical category, were obtained from the unit's database, a system independent of the protocol management system. Data on the clinical chemistry tests requested for each of these patients were obtained from the laboratory computer system. Since the laboratory system only provides information to the protocol management system and does not receive any data from it the sources used for the patient data and for the investigation data should both be totally unaffected by the introduction of the protocol management system.

ANALYSIS

Total use of laboratory services and out of hours use were analysed in terms of the number of tests per patient day, for the unit overall and for each category of patient because of a strong linear relation (correlation coefficient 0.89 ) between the number of investigations requested for patients and the length of bed stay of patients. Considering the number of tests per patient day effectively ignores any variation in usage which is merely reflecting a change in the mean bed stay.

Direct costs of laboratory tests were calculated by the method of Broughton and Hogan $^{6}$ and used to examine the effect of the system on laboratory expenditure. Comparisons between the periods before and after the introduction of the system were made using two-sample Student's $t$ tests or MannWhitney U tests, as appropriate. Since there was an investigation protocol for each category of patient admitted to the unit no subset of patients could be used as a meaningful control group.

Although it was possible to assess in some detail the effect of the protocol management system on the clinical chemistry requests of the unit, no attempt was made to detect any change in patient outcome due to the introduction of the system: although survival rate could have been used as an outcome measure for patients undergoing transplantation, their clinical states varied to such a degree that thousands of patients would have been required to detect any significant effect; for many of the other patient categories (for example, initial assessment) there was no obvious outcome measure.

The total number of results received by the system for patients in the year after its introduction was obtained from the system, as was the total number of these investigations proposed by the protocols: these figures were used to calculate an overall measure of "protocol performance."

\section{Results}

USAGE AND EXPENDITURE

Tables 1 and 2 show the number of patients admitted to the unit, their mean bed stay, and details of the clinical chemistry usage per patient and per patient day and direct expenditure per patient day for each clinical category before and after the introduction of the computerised protocol management system. Overall, the total number of tests requested per patient day declined significantly, by $17 \%$ ( $p<0.001$, Student's $t$ test; table 2). In addition, the number of out of hours tests requested per patient day fell from 0.31 to 0.16 , a $48 \%$ reduction ( $p<0.001$; MannWhitney $U$ test). Use of the system therefore reduced the total clinical chemistry requesting per patient day and greatly reduced the much more expensive out of hours requesting, resulting in a significant overall reduction in direct laboratory costs of $28 \%(p<0.001$, Mann-Whitney U test; table 1).

Table 3 shows for the periods before and after the introduction of the system the number of requests per patient day for urea and electrolyte tests (creatinine, potassium, sodium, and urea tests), liver function tests, bone profile (albumin, alkaline phosphatase, calcium, and total protein tests), calcium tests and others (that is, none of the above). Although part of the bone profile, calcium is shown separately since there was an immediate and sustained reduction in the number of bone profiles requested whereas the number of requests for calcium measurements per patient day fell by $50 \%$ after the introduction of the system and by a further $90 \%$ after the change in protocols.

Table 1 Number (percentage) of patients, bed stay, and direct costs of clinical chemistry investigations for each category of patient one year before and after introduction of computerised protocol management system

\begin{tabular}{|c|c|c|c|c|c|c|c|c|c|c|}
\hline \multirow[t]{2}{*}{ Category of patient } & \multicolumn{2}{|c|}{ No (\%) of patients } & \multicolumn{4}{|c|}{ Bed stay per patient (days) } & \multicolumn{4}{|c|}{ Direct costs per patient day $(£)$} \\
\hline & Before & After & Before $†$ & After & $\%$ Change & $\begin{array}{l}\text { Mann- } \\
\text { Whitney } \\
\text { statistic }\end{array}$ & Before $†$ & After $†$ & $\%$ Change & $\begin{array}{l}\text { Mann- } \\
\text { Whitney } \\
\text { statistic }\end{array}$ \\
\hline Initial assessment & $177(27)$ & $153(18)$ & $7 \cdot 5(4-12)$ & $10 \cdot 0(5-15)$ & +33 & $3 \cdot 04^{\star}$ & $2 \cdot 54(1 \cdot 37-4 \cdot 61)$ & $2 \cdot 46(1 \cdot 54-3 \cdot 83)$ & -3 & $0 \cdot 21$ \\
\hline Reassessment (routine) & $33(5)$ & $67(8)$ & $6 \cdot 0(4-9)$ & $5 \cdot 0(4-10)$ & -17 & $0 \cdot 14$ & $0.35(0.21-0.94)$ & $0.76(0.21-1.94)$ & +117 & $1 \cdot 38$ \\
\hline Reassessment (problem) & $30(5)$ & $45(5)$ & $16 \cdot 0(5-30)$ & $8 \cdot 0(5-13)$ & -50 & $2 \cdot 41^{\star}$ & $1 \cdot 67(1 \cdot 21-2 \cdot 25)$ & $0 \cdot 83(0 \cdot 38-1 \cdot 23)$ & -50 & $3 \cdot 24^{\star \star}$ \\
\hline Transplant & $106(16)$ & $112(13)$ & $20 \cdot 0(6-24)$ & $18 \cdot 0(6-25)$ & -10 & 0.58 & $2 \cdot 84(1 \cdot 94-4 \cdot 18)$ & $2 \cdot 51(1 \cdot 86-3 \cdot 42)$ & -12 & $1 \cdot 46$ \\
\hline Post-transplant (problem) & $84(13)$ & $112(13)$ & $7 \cdot 5(3-14)$ & $6 \cdot 5(3-14)$ & -13 & $0 \cdot 31$ & $1 \cdot 65(1.03-2 \cdot 37)$ & $1.43(0.78-1.90)$ & -13 & $2 \cdot 44^{\star}$ \\
\hline $\begin{array}{l}\text { Post-transplant } \\
\text { (annual review) }\end{array}$ & $86(13)$ & $146(18)$ & $2 \cdot 0(2-3)$ & $3 \cdot 0(2-3)$ & +50 & $1 \cdot 87$ & $1 \cdot 29(0 \cdot 91-1 \cdot 43)$ & $0 \cdot 86(0 \cdot 74-1 \cdot 29)$ & -33 & $5 \cdot 50^{\star \star \star}$ \\
\hline $\begin{array}{l}\text { Emergency } \\
\text { (acute hepatic failure) }\end{array}$ & $39(6)$ & $62(7)$ & $4 \cdot 0(3-5)$ & $4 \cdot 5(3-7)$ & +13 & $1 \cdot 21$ & $2 \cdot 34(0 \cdot 91-3 \cdot 77)$ & $1 \cdot 68(0.60-2 \cdot 56)$ & -28 & $1 \cdot 74$ \\
\hline $\begin{array}{l}\text { Emergency } \\
\quad \text { (acute problem - } \\
\text { chronic disease) }\end{array}$ & $32(5)$ & $19(2)$ & $13 \cdot 5(3-21)$ & $10 \cdot 0(4-16)$ & -26 & 0.91 & $2 \cdot 92(1.93-5 \cdot 59)$ & $2 \cdot 44(0.92-3 \cdot 49)$ & -16 & $1 \cdot 20$ \\
\hline Other & $26(4)$ & $69(8)$ & $1 \cdot 0(1-5)$ & $2 \cdot 0(1-3)$ & +100 & $0 \cdot 20$ & $0 \cdot 00(0 \cdot 00-1 \cdot 16)$ & $0 \cdot 12(0 \cdot 00-1 \cdot 19)$ & & $0 \cdot 10$ \\
\hline Overall & $654(100)$ & $833(100)$ & $5 \cdot 0(3-15)$ & $5 \cdot 0(3-12)$ & 0 & 1.09 & $1 \cdot 79(0 \cdot 94-2 \cdot 96)$ & $1 \cdot 29(0 \cdot 71-2 \cdot 37)$ & -28 & $6 \cdot 86^{\star \star \star}$ \\
\hline
\end{tabular}

+ Median (interquartile range) values.
${ }^{\star} \mathrm{p}<0.05,{ }^{\star \star} \mathrm{p}<0.01,{ }^{\star \star \star} \mathrm{p}<0.001$. 
Table 2 Total clinical chemistry usage for each category of patient one year before and after introduction of computerised protocol management svitem

\begin{tabular}{|c|c|c|c|c|c|c|c|c|}
\hline \multirow[t]{2}{*}{ Categom of patient } & \multicolumn{4}{|c|}{ Total tests per patient } & \multicolumn{4}{|c|}{ Total tests por pationt day } \\
\hline & Beforet & Aftert & " Changc & $\begin{array}{c}\text { Mann-Whitney } \\
\text { statistic }\end{array}$ & Beforet & Afict: & " Chamger & $\begin{array}{l}\text { Student } \\
\text { tstatistic }\end{array}$ \\
\hline Initial assessment & $48 \cdot 0(33-73)$ & $45 \cdot 0(31-81)$ & -6 & $0 \cdot 10$ & $7 \cdot 1(2 \cdot 9)$ & $5 \cdot+(3 \cdot 0)$ & 25 & $5 \cdot 23^{\star \star \star \star}$ \\
\hline Reassessment (routine) & $22 \cdot 0(1437)$ & $22 \cdot 0(15-32)$ & 0 & 0.26 & $4 \cdot 8(2.7)$ & $3.7(2.7)$ & 22 & 1.92 \\
\hline Reassessment (problem) & $120 \cdot 0(57-209)$ & $46 \cdot 0(24-70)$ & -62 & $4 \cdot 04^{\star \star \star}$ & $7 \cdot 7(2 \cdot 1)$ & $0.3(2 \cdot 3)$ & $\overline{10}$ & $2.6 \overline{7} \star \star \star$ \\
\hline Transplant & $203 \cdot 0(45-270)$ & $175.5(50-253)$ & -14 & $1 \cdot 28$ & $11 \cdot 0(2 \cdot 8)$ & $9 \cdot 6(3 \cdot 3)$ & 13 & $-3.37 \star \star \star \star$ \\
\hline Post-transplant (problem) & $56 \cdot 5(24-114)$ & $41.5(20-88)$ & 27 & 1.39 & $7 \cdot 8(2 \cdot 5)$ & $6.9(2.3)$ & 11 & $2 \cdot 61^{\star \star}$ \\
\hline Post-transplant (t tube removal) & $16 \cdot 5(11-19)$ & $17 \cdot 5(9-22)$ & +6 & 0.27 & $6 \cdot 6(4 \cdot 0)$ & $5 \cdot 0(2 \cdot 1)$ & 25 & $2 \cdot 41^{\star}$ \\
\hline Post-transplant (annual review) & $18 \cdot 0(15-20)$ & $18 \cdot 5(15-22)$ & +3 & $0 \cdot 49$ & $7 \cdot 4(4 \cdot 1)$ & $6 \cdot 6(2 \cdot 9)$ & 12 & 1.73 \\
\hline Emergency (acute hepatic failure) & $24 \cdot 5(21-49)$ & $40 \cdot 0(21-66)$ & +63 & $1 \cdot+1$ & $6 \cdot 7(3 \cdot 8)$ & $7 \cdot 8(4 \cdot 0)$ & +17 & 1.37 \\
\hline Emergency (acute problem - chronic disease) & $145 \cdot 0(26-191)$ & $71 \cdot 0(31-154)$ & 51 & 1.39 & $11 \cdot 1(+2)$ & $8 \cdot 0(+1)$ & 28 & $2 \cdot 57 *$ \\
\hline Other & $0 \cdot 0(0-45)$ & $8 \cdot 0(0-16)$ & & $0 \cdot 04$ & $6 \cdot 2(4 \cdot 3)$ & $5 \cdot 5(4 \cdot 1)$ & 11 & $(0.73$ \\
\hline Overall & $38 \cdot 0(18 \quad 114)$ & $29 \cdot 0(16-72)$ & 24 & $4 \cdot 03^{\star \star \star}$ & $8 \cdot 5(3 \cdot 6)$ & $7 \cdot 0(3 \cdot 5)$ & 17 & $8 \cdot 10^{\star \star \star}$ \\
\hline
\end{tabular}

†Median (interquartile range) values.

$\ddagger$ Mean (standard deviation) values

${ }^{\star} \mathrm{p}<0 \cdot 05,{ }^{\star \star} \mathrm{p}<0 \cdot 01,{ }^{\star \star \star} \mathrm{p}<0 \cdot 001$

It is evident from tables 2 and 3 that a large part of the overall reduction in clinical chemistry usage was due to substantial decreases in the numbers of requests for bone profiles and calcium measurements. However, even if both these investigations are excluded from the analysis there are still significant reductions in usage overall, with the number of tests requested per patient decreasing by $16 \%$ and the number of tests per patient-day decreasing by $5 \%$.

PROTOCOL PIERFORMANCE

Figure 1 shows that for 10 of the less common tests the degree of compliance with the existing protocols increased significantly after introduction of the system. Compliance was calculated as the percentage of those patients requiring a particular investigation (according to the protocols) who were actually tested. It was less than $100 \%$ because some investigations proposed by the system were not done, as results from other hospitals were already available. In addition, a comparison of patients' classifications on the system with their notes disclosed that some patients had been incorrectly classified.

Improved compliance resulted in an increase in the usage of some of the less common tests, which generally tend to have higher unit costs. Most of the tests in figure 1 were requested for patients undergoing initial assessment, which explains why, for this category, a $25 \%$ decrease in the total number of tests requested per patient day (table 2 ) resulted in only a 3\% decrease in direct laboratory expenditure per patient day (table 1).

In the year after its introduction the system received 60546 results of clinical chemistry investigations on inpatients in the unit, $50367(83 \%)$ of which were proposed by the protocol management system, which is one measure of protocol performance. Deter-

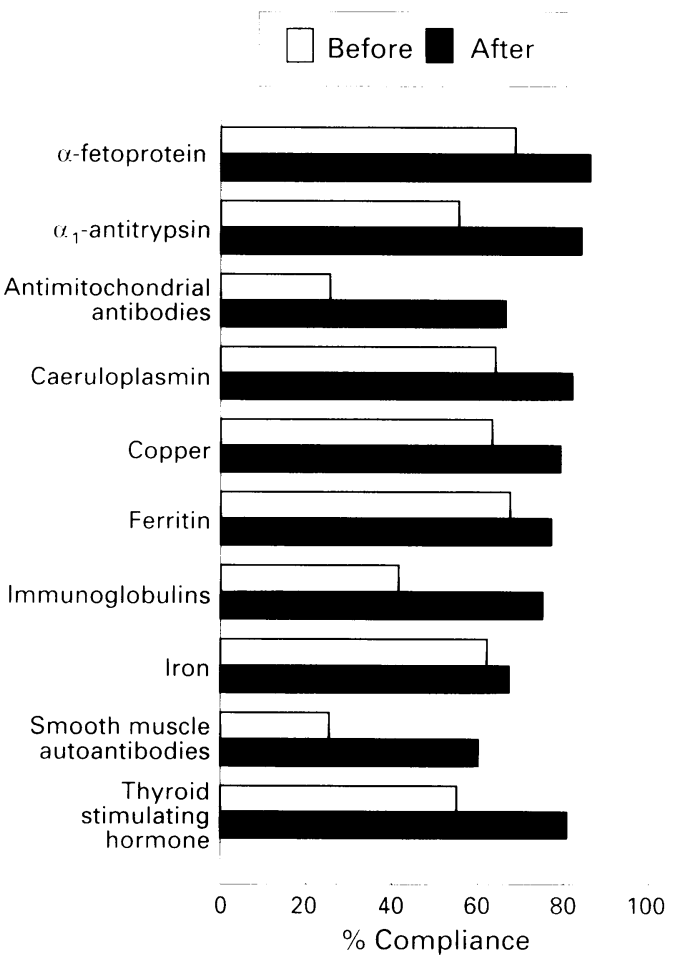

Figure 1 Compliance for 10 less commonly requested tests one vear before and after introduction of computerised protocol management sistem

mining how many investigations the protocols would have proposed if they had been followed exactly was not possible, as if a test proposed by the protocol is not requested there is no way of knowing whether the result would have generated another proposal (through a dynamic rule - see appendix).

VARIATION

Although the investigation protocols contained in the system provide a structured approach to laboratory requesting, the distribution of the mean number of clinical

Table 3 Numbers of clinical chemistry requests per patient day one vear before and after introduction of computerised protocol management system

\begin{tabular}{|c|c|c|c|c|}
\hline Requests & Beforet & Aftert & " Change & Mann-Whitney statisti \\
\hline Plasma urea and electrolytes & $0.67(0.42-0.95)$ & $0.56(0.33-0.83)$ & 16 & $2 \cdot 32 \star$ \\
\hline Liver function tests & $0.60(0.39-0.88)$ & $0.50(0.33-0.80)$ & 17 & 1.92 \\
\hline Bone profile & $0.40(0.17-0.67)$ & $(0.00(0.00-0.10)$ & 100 & $18 \cdot 4^{\star \star \star}$ \\
\hline Calcium & $0.50(0.33-0.72)$ & $0.07(0.00-0.25)$ & 86 & $16 \cdot 3^{\star \star \star}$ \\
\hline Others & $1.27(0.50-2.67)$ & $1.38(0.33-3.14)$ & +9 & 0.32 \\
\hline
\end{tabular}

†Median (interquartile range) values.

${ }^{\star} \mathrm{p}<0.05,{ }^{\star \star \star} \mathrm{p}<0 \cdot 001$ 


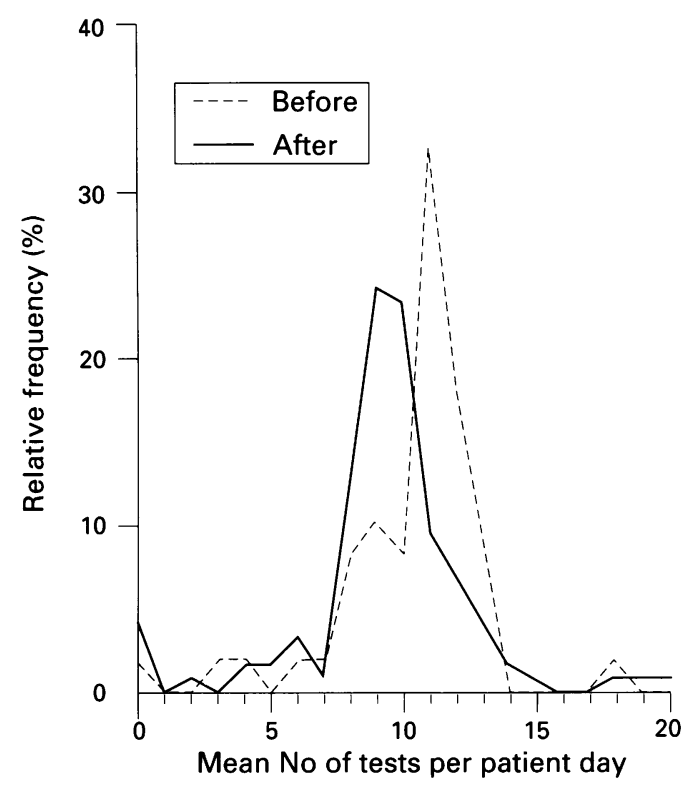

Figure 2 Distribution of mean number of tests per patient day for patients in transplant category, one year before and after introduction of computerised protocol management system

chemistry tests per patient day for patients in the "transplant" category before and after the introduction of the system (fig 2) displays greater variation between patients (coefficient of variation $26 \%$ before and $33 \%$ after introduction of the system). This suggests that this system is flexible enough to allow more detailed investigation of those patients who require it. Much of this flexibility arises from the dynamic protocols, since the frequency with which some tests are requested is dependent on previous results (see appendix); the remainder is due to the freedom of the house officers to ask for additional investigations if required $(17 \%$ of all tests were requested in this way).

\section{Discussion}

The potential advantages of computerised decision support systems have long been known, ${ }^{7-9}$ but currently there are few such systems in routine use. In our study the introduction of the computerised protocol management system reduced both total and out of hours clinical chemistry requesting per patient day, resulting in an overall reduction in direct laboratory costs (table 1). However the purpose of this study was not to cut costs but to promote more appropriate use of laboratory services.

A computerised protocol management system can only be as good as the protocols it contains, and clinicians in other hospitals might regard some of the investigation protocols used in this study as inappropriate. However, if an investigation is defined as (locally) appropriate if it is one required by the protocols (that is, deemed by the senior clinicians to be appropriate), then the system corrected not only overutilisation (as shown by the overall reduction in usage (table 2)) but also underutilisation of the laboratory (fig 1). Clearly scope still exists for improving the appropriateness of laboratory testing, perhaps by refinement of the existing protocols and certainly through the implementation of more specific protocols.

This system is a tool to be used by the house officers in the course of their normal duties and is designed to alleviate the drudgery of some of their more mundane tasks. It does not remove or diminish the house officers' role in the laboratory investigation of their patients since they are able at any time to request a set of investigations which is different from that suggested by the system. However, the monthly feedback provided to the consultants enables the requesting patterns of junior staff to be monitored closely. The senior clinicians discuss any regular and systematic deviations from the protocol with their house officers, thus educating them in the appropriate use of laboratory services.

The efficacy of the protocols can be judged by measures of protocol performance (for example $83 \%$ of all tests requested were proposed by the protocols), although it is not possible to state a standard level of acceptability with which to compare such measures. However, if the protocol performance were poor little or no time saving would be achieved through using the system and the monthly feedback would show systematic deviations from the protocols. Since neither of these is true for this system, and accepting that there will always be the need to perform additional tests on very sick patients, the protocols used seem to be performing well.

The introduction of the computerised protocol management system has several implications for the quality of patient care: patients are more likely to be investigated in a manner deemed appropriate by the consultant, which will improve the information available, enabling better decisions on patient management; resources are used more appropriately and therefore more effectively; and the decreased amount of time spent by the doctor performing routine tasks allows more time for medical activity.

The system described here was introduced into a specialist unit and was concerned only with pathological investigations. However, a similar approach could be adopted in any setting in which investigation protocols pertain. In particular, there is a need to extend the scope of the system to include other laboratory services such as radiology and to implement the system in other clinical settings. Indeed a second system has been in routine operation in another specialist unit ${ }^{10}$ for over a year and a further two have just been introduced into general medical settings. As more hospitals begin to use these systems there will be the potential to compare different protocols for the same clinical conditions with a view to establishing a wider consensus on appropriate investigation. We believe that computerised decision support systems such as these provide a much more structured approach to investigation and therefore will play a major part in the health care process. 


\section{Appendix}

\section{Examples of investigation protocols}

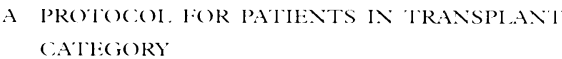

Static rules

The following investigations will be proposed: Liver function tests on days 1 to 16 and every three days thereafter

Full blood count on davs 1 to 16 and every three days thereafter

Prothrombin time on davs 1 to 16 and every three days thereafter

Urea and electrolytes on davs $1,2,3,4,5,6$, $8,10,13$ and every three days thereafter Cyclosporin every day

Magnesium on days 2, 9, 16

\section{Dynamic rules}

(All rules apply to latest results or, in the case of differences between results, to the last two results.)

If potassium $>5.5 \mathrm{mmol} / \mathrm{l}$ or $<3 \mathrm{mmol} / \mathrm{l}$ propose urea and electrolyte tests today If potassium $>5.1 \mathrm{mmol} / \mathrm{l}$ or $<3.4 \mathrm{mmol} / \mathrm{l}$ or has changed by more than $0.4 \mathrm{mmol} / \mathrm{l}$, or if urea $>20 \mathrm{mmol} / \mathrm{l}$ or (if day $<11$ and urea $>10$ $\mathrm{mmol} / \mathrm{l}$ ) or if urea has changed by more than 4 $\mathrm{mmol} / \mathrm{l}$, or if creatinine $>210 \mu \mathrm{mol} / \mathrm{l}$ or (if day $<11$ and creatinine $>110 \mu \mathrm{mol} / \mathrm{l}$ ) or if creatinine has changed by more than $25 \mu \mathrm{mol} /$ 1, propose urea and electrolyte tests tomorrow If urea $>15 \mathrm{mmol} / \mathrm{l}$, or if creatinine $>160$ $\mathrm{mmol} / \mathrm{l}$, propose urea and electrolyte tests for the day after tomorrow, unless above rule is proposing urea and electrolytes tomorrow If albumin $<20 \mathrm{~g} / \mathrm{l}$, or if bilirubin $>50 \mu \mathrm{mol} / \mathrm{l}$ or has increased by more than $10 \mu \mathrm{mol} / \mathrm{l}$, or if aspartate aminotransferase $>50 \mathrm{iu} / \mathrm{l}$ or has increased by more than $10 \mathrm{iu} / \mathrm{l}$, or if alkaline phosphatase $>400 \mathrm{iu} / \mathrm{l}$, propose liver function tests tomorrow

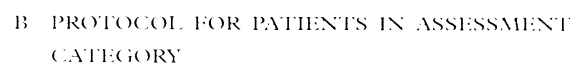

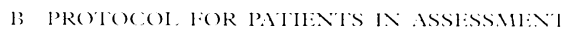
(ATISCORY

(All the rules for this category are static rules)

The following investigations to be proposed on day 2:

Liver function tests

Full blood count

Prothrombin time

Urea and electrolyte tests

Ionised calcium

Total protein

Creatinine clearance

Blood gases

Blood grouping

Alphafetoprotein

Alpha ${ }_{1}$-antitrypsin

Antimitochondrial antibodies

Antinuclear antibodies

Caeruloplasmin

Copper

Ferritin

Immunoglobulins

Iron

Smooth muscle autoantibodies

Thyroid stimulating hormone

Transferrin

The following investigations to be proposed on day 8 and every seven davs thereafter:

Liver function tests

Full blood count

Prothrombin time

Urea and electrolytes
PGN and MP acknowledge financial support from the Department of Health and the EEC AIM OPENLABS Project.

1 Fraser CG, Woodford FP. Strategies to modify the testrequesting patterns of clinicians. Ann Clin Biochem requesting pattern

2 Blery C, Charpak Y, Szatan M, Darne B, Fourgeaux B, Chastang CI, it al. Evaluation of a protocol for selective ordering of preoperative tests. Lancet 1986; ; $139-41$

3 Mutimer D, McCauley B, Nightingale P, Ryan M, Peters M, Neuberger J. Computerised protocols for laboratory investigation and their effect on use of medical time and resources. 7 Clin Pathol 1992;45:572-4.

4 McCauley BA, Parekh J, Clark IR. A MUMPS application of rule based processing. In: Dayhoff RE, ed. Proceedings of the MUMPS Users' Groun Meeting: 1991; New Orleans. MUMPS Users Mroup, 1991:18-21.
5 Peters M, Clark IR, Parekh J, McCauley BA, Ryan MF, Neuberger JM, ct al. Automatic application of rule-based decision support; a specialist unit investigation manager. In: Richards B, ed. Ciurrent perspectious in healthicar computing. Weybridge, Surrev: British Healtheare Computing, 1991:129-36.

6 Broughton PMG, Hogan TC. A new approach to the costing of clinical laboratory tests. Ann (ilin Biochem 1981:18:330 12.

MeDonald CJ. Use of a computer to detect and respond to clinical events: its effect on clinician behavior. Am Intem Med 1976;84:162-7.

Young DW. An aid to reducing unnecessarv investigations. B.179 1980;281:1610-1

) Young DW. A surver of decision aids for clinicians. B.MY 1982;285:1332-6.

10 Peters M, Clark IR, McCauley BA, Rvan .MF, Parckh J, King JA. Protocol management systems - laboratory investigation of renal patients. In: Richards B, ed. Current perspectices in healthcare computing. Weybridge, Surrey: British Healthcare Computing 1092.20612 